\title{
Aspectos clínicos da síndrome de Asperger na comunidade de uma universidade
}

\section{pública}

\author{
Clinical aspects of Asperger syndrome in the Community of a public university \\ Aspectos clínicos da síndrome de Asperger na comunidade de uma universidade pública
}

Recebido: 25/07/2021 | Revisado: 29/07/2021 | Aceito: 29/07/2021 | Publicado: 05/08/2021

\author{
Anteia Galati Acioli Lindoso \\ ORCID https://orcid.org/0000-0002-6763-3801 \\ Universidade Federal de São João del-Rei, Brasil \\ E-mail:anteialindoso@gmail.com \\ Nicaelen Sobrinho \\ ORCID https://orcid.org/0000-0002-8635-2917 \\ Universidade Federal de São João del-Rei, Brasil \\ E-mail: nicaelensobrinho@ufsj.edu.br \\ Rafael de Avila Justino da Silva \\ ORCID https://orcid.org/0000-0003-4075-3798 \\ Universidade Federal de São João del-Rei, Brasil \\ E-mail: Unfaelbankai@gmail.com \\ Ana Catarina de Almeida Pinho Ferreira \\ ORCID https://orcid.org/0000-0001-6362-6770 \\ Universidade Federal de São João del-Rei, Brasil \\ Centro Universitário Presidente Tancredo de Almeida Neves, Brasil \\ E-mail: catarinaana@ufsj.edu.br \\ Sheila Ferreira Miranda \\ ORCID https://orcid.org/0000-0002-3144-6254 \\ Universidade Federal de São João del-Rei, Brasil \\ E-mail: sheilaze@gmail.com \\ Laila Cristina Moreira Damázio \\ ORCID https://orcid.org/0000-0001-7370-8892 \\ Universidade Federal de São João del-Rei, Brasil \\ Centro Universitário Presidente Tancredo de Almeida Neves, Brasil \\ E-mail: lailadamazio@ufsj.edu.br
}

\begin{abstract}
Resumo
A Síndrome de Asperger (SA) é um transtorno do neurodesenvolvimento que cursa com deterioração significativa nas áreas sociais, ocupacionais e outras importantes atividades do indivíduo. Os portadores da síndrome, geralmente apresentam habilidades em memorização e são bons em matemática ou ciência. A SA é uma doença recente, onde ainda existem controvérsias sobre o diagnóstico e tratamento, tendendo a ser subdiagnosticada. Objetivo: Avaliar a presença dos aspectos clínicos da SA entre estudantes, técnicos e docentes de uma universidade federal do interior de Minas Gerais, Brasil. Metodologia: A Escala Australiana para SA foi aplicada através de um questionário online e os dados dos escores foram analisados pelo teste ANOVA e o teste de correlação de Pearson, utilizando o programa estatístico GraphPrism 5.0, considerando um nível de significância de $p<0,05$. Resultados: O estudo permitiu evidenciar uma baixa incidência de aspectos clínicos da SA entre os docentes, discentes e técnicos da instituição, sendo que, maiores escores da escala foram encontradas entre os discentes e docentes das áreas de exatas e humanas, e os menores na área da saúde. Conclusão: A população estudada apresentou poucos aspectos clínicos para SA já que os escores mais baixos foram mais prevalentes entre os indivíduos.
\end{abstract}

Palavras-chave: Estudantes; Faculdade; Síndrome de Asperger.

\begin{abstract}
Asperger Syndrome (AS) is a neurodevelopmental disorder that progresses with significant deterioration in the individual's social, occupational and other important activities. People with the syndrome usually have memorization skills and are good at math or science. AS is a recent disease, where there is still controversy about diagnosis and treatment, tending to be underdiagnosed. Objective: To evaluate the presence of clinical aspects of AS among students, technicians and professors at a federal university in the interior of Minas Gerais, Brazil. Methodology: The Australian Scale for AS was applied through an online questionnaire and the score data were analyzed by the ANOVA test and the Pearson correlation test, using the GraphPrism 5.0 statistical program, considering a significance level of $p<0.05$. Results: The study showed a low incidence of clinical aspects of AS among the institution's professors, students and technicians, with higher scale scores being found among students and professors in the
\end{abstract}


sciences and humanities, and the smallest in the field. of health. Conclusion: The population studied showed few clinical aspects for AS since the lowest scores were more prevalent among individuals.

Keywords: Students; Faculty; Asperger Syndrome.

\section{Resumen}

El síndrome de Asperger (AS) es un trastorno del desarrollo neurológico que progresa con un deterioro significativo en las actividades sociales, ocupacionales y otras actividades importantes del individuo. Las personas con el síndrome generalmente tienen habilidades de memorización y son buenas en matemáticas o ciencias. La EA es una enfermedad reciente, en la que aún existe controversia sobre el diagnóstico y tratamiento, con tendencia a estar infradiagnosticada. Objetivo: Evaluar la presencia de aspectos clínicos de EA entre estudiantes, técnicos y profesores de una universidad federal del interior de Minas Gerais, Brasil. Metodología: Se aplicó la Escala Australiana para AS a través de un cuestionario online y los datos de las puntuaciones se analizaron mediante la prueba ANOVA y la prueba de correlación de Pearson, utilizando el programa estadístico GraphPrism 5.0, considerando un nivel de significancia de p <0.05. Resultados: El estudio mostró una baja incidencia de aspectos clínicos de EA entre los profesores, estudiantes y técnicos de la institución, encontrándose puntuaciones más altas en las escalas de estudiantes y profesores de ciencias y humanidades, y las más pequeñas en el campo de la salud. Conclusión: La población estudiada mostró pocos aspectos clínicos para la EA ya que los puntajes más bajos fueron más prevalentes entre los individuos.

Palabras clave: Estudiantes; Facultad; Síndrome de Asperger.

\section{Introdução}

A SA de acordo com o Manual Diagnóstico e Estatístico de Transtornos Mentais - Diagnosticand Statistical Manual of Mental Disorders (DSM), é um transtorno do neurodesenvolvimento que faz parte do Transtorno do Espectro Autista (TEA), junto com o Transtorno Autista, o Transtorno Desintegrativo na Infância, o Transtorno de Rett e o Transtorno Global do Desenvolvimento. O TEA é caracterizado por déficits na comunicação e interação social, além de padrões repetitivos e restritos de comportamentos, interesses e atividades (Diagn, 2013). Como diferencial, a SA não costuma apresentar comprometimento linguístico ou intelectual (Teodoro et al., 2013). Acredita-se que sua origem seja principalmente genética, podendo, na maioria das vezes, serem identificadas características da síndrome em parentes de primeiro e segundo grau, sendo os fatores genéticos mais comuns na SA do que no Autismo clássico (Eckel et al., 2015).

A SA foi diagnosticada pela primeira vez em 1944, pelo pediatra Hans Asperger. Com grande interesse em educação especial, e sem conhecimento dos estudos de Leo Kanner sobre o autismo, Asperger estudou quatro de seus pacientes que tinham a dificuldade de interagir socialmente. Denominou a condição por ele descrita como "psicopatia aurística", indicando um transtorno estável de personalidade marcado pelo isolamento social (Teodoro et al., 2013). A Síndrome de Asperger somente foi reconhecida em 1994, logo após a publicação da Classificação Internacional de Doenças (CID-10) e do DSM (Dias, 2015; Eckel et al., 2015).

Essa síndrome já foi diagnosticada erroneamente como uma perturbação obsessiva - compulsiva, depressão e até esquizofrenia. Os pacientes com SA não apresentam atrasos significativos no desenvolvimento cognitivo e algumas vezes passam a vida sendo estigmatizadas por seus hábitos não usuais em relação aos padrões de comportamento considerados adequados para a sociedade (Dias, 2015).

As crianças com SA apresentam uma linguagem bem desenvolvida do ponto de vista gramatical, dificultando o diagnóstico precoce. Apesar disso, tendem a não compreender a linguagem não-verbal, que inclui gestos e expressões físicas e as informações implícitas ou conotativas da comunicação, dificultando as interações sociais (Eckel et al., 2015; Franz e Dawson, 2019). Podem ainda apresentar graus de comprometimento social, principalmente devido aos comportamentos não convencionais e movimentos repetitivos. Alguns autores consideram a SA como um tipo de autismo de alto funcionamento, embora existam diferenças nas características operacionais clínicas, devido a presença de problemas de linguagens típicos. Por existir variação no nível da gravidade da SA, os indivíduos que são ligeiramente afetados muitas vezes nem chegam a ser diagnosticados (Brito Brito et al., 2011). 
Os estudos sugerem que a SA é mais comum que o autismo clássico, sendo encontrada numa taxa de 2 a 4 de 10.000 crianças, mas devido à ausência de definições diagnósticas esses valores tendem a ser subestimados podendo chegar a 1:1000 (Klin, 2006). Existem ainda poucos estudos sobre a síndrome e os questionamentos sobre a sua frequência e os fatores de risco ainda são controversos, no entanto, estudos demonstram que a cada 10 pacientes com a SA, 9 são homens (Klin, 2006).

O diagnóstico da SA, de acordo com o DSM-V, inclui mudanças qualitativas nas relações sociais, padrões de comportamento, interesses restritivos e repetitivos, assim como atividades estereotipadas. É geralmente identificada no início da fase escolar, podendo ser diagnosticada como autismo na pré-escola (Eckel et al., 2015). Isso aponta a necessidade de serem revistos os diagnósticos de autismo feitos nessa fase, além da necessidade de instrumentos apropriados para avaliação comportamental e cognitiva.

A escassez de estudos e métodos diagnósticos específicos voltados para a avaliação de indivíduos adultos com suspeita de SA contribuiu para realização desse estudo que propõe investigar a presença de aspectos clínicos da SA em alunos, professores e técnicos de uma universidade federal do interior de Minas gerais/Brasil.

\section{Metodologia}

A presente pesquisa foi realizada entre os meses de junho a dezembro de 2018 nos três Campi da sede de uma Universidade Federal no interior de Minas Gerais/Brasil; na qual o público participante foi separado nas categorias de docentes, técnicos e discentes. O estudo é original, quantitativo com delineamento transversal (Estrela; Pereira, 2018) visando avaliar a presença dos aspectos clínicos da SA entre os representantes das categorias.

Para a realização deste estudo foi utilizada a Escala Australiana para SA, validada em português por Caires (2012) e adaptada pelos pesquisadores do presente projeto. Esse questionário é utilizado na primeira fase da obtenção do diagnóstico de SA e avalia a capacidade de comunicação, concentração, desenvolvimento cognitivo e as habilidades sociais presentes ou não durante a infância através de 34 questões, onde cada pergunta é pontuada pelo participante em uma escala de zero a seis, de acordo com a frequência em que os fatos apresentados ocorriam na infância, podendo totalizar de 0 a 204 pontos no escore final do questionário. Os escores da escala foram separados por intervalos de 0-39, 40-79, 80-119, 120-159 e 160-204 para análise da gravidade e o número de aspectos clínicos presentes nas categorias avaliadas.

A escala modificada foi encaminhada através de um formulário online enviado ao e-mail de todos os professores, técnicos e alunos dos três Campi da sede da universidade, acima de 18 anos e que concordaram em participar da pesquisa e assinaram eletronicamente o termo de Consentimento Livre e Esclarecido presente na página inicial do documento. O tempo total para preenchimento do questionário foi de 10 minutos, possuindo apenas questões objetivas. Optou-se por não enviar aos participantes o escore total obtido ao final do preenchimento, pois o mesmo não possui valores pré-determinados para o diagnóstico da SA, uma vez que é usado apenas na primeira fase da obtenção do diagnóstico, necessitando de uma avaliação profissional mais específica em relação aos aspectos que se sobressaíram.

A população de docentes e discentes foi distribuída entre as áreas das ciências humanas, exatas, naturais e da saúde, de acordo com as Tabelas 1, 2 e 3. 
Tabela 1. Número de professores por áreas em cada departamento da universidade federal do interior de Minas Gerais

\begin{tabular}{lllll}
\hline Ciências Humanas & Ciências Exatas & Ciências Naturais & $\begin{array}{l}\text { Ciências } \\
\text { Saúde }\end{array}$ & da \\
\hline 113 professores & 156 professores & 63 professores & 69 professores \\
\hline
\end{tabular}

Fonte: Pró-Reitoria de Gestão e Desenvolvimento de Pessoas - Universidade Federal do Interior de Minas Gerais

Tabela 2. Número de discentes por áreas em cada curso da universidade federal do interior de Minas Gerais.

\begin{tabular}{|c|c|c|c|}
\hline Ciências Humanas & Ciências Exatas & Ciências Naturais & Ciências da Saúde \\
\hline Letras (201) & Geografia (199) & $\begin{array}{l}\text { Ciências Biológicas } \\
(210)\end{array}$ & $\begin{array}{l}\text { Educação Física } \\
(134)\end{array}$ \\
\hline Filosofia (196) & Engenharia Mecânica (1040) & Quimica (197) & Psicologia (368) \\
\hline Pedagogia (185) & Engenharia Elétrica (816) & Física (122) & Medicina (113) \\
\hline História (163) & Matemática (105) & Zootecnia (344) & \\
\hline Música (182) & Engenharia de Produção (332) & & \\
\hline Comunicação Social (204) & Administração (330) & & \\
\hline \multirow[t]{5}{*}{ Teatro (181) } & Ciências Contábeis (160) & & \\
\hline & Ciências Econômicas (334) & & \\
\hline & Arquitetura e Urbanismo (333) & & \\
\hline & Artes Aplicadas (113) & & \\
\hline & Ciência da Computação (262) & & \\
\hline 1312 alunos & 4024 alunos & 873 alunos & 615 alunos \\
\hline
\end{tabular}

Fonte: Divisão de Controle Acadêmico - Universidade Federal do Interior de Minas Gerais

Os técnicos não foram divididos por áreas devido à existência de diversos campos de atuação dentro da universidade, sendo as análises deste grupo baseadas apenas no número total e na comparação com a totalidade de professores e alunos.

Tabela 3. Número de técnicos em cada Campus da Sede da Universidade Federal do Interior de Minas Gerais

\begin{tabular}{lll}
\hline \multicolumn{1}{c}{ Campus I } & Campus II & Campus III \\
\hline 66 técnicos & 284 técnicos & 27 técnicos \\
& & \\
\hline
\end{tabular}

Fonte: Pró-Reitoria de Gestão e Desenvolvimento de Pessoas - Universidade Federal do Interior de Minas Gerais

Para o cálculo amostral foi considerado um erro de 5\% e nível de confiança de $95 \%$ da população total de 7.629 participantes distribuídos entre professores, técnicos e estudantes. Dessa forma, o número total de participantes da pesquisa para representação estatística da população estudada foi de 198 participantes.

A coleta de dados através do questionário online tem se popularizado cada vez mais, sendo uma de suas principais vantagens a praticidade na seleção da amostra sem que seja necessário o deslocamento dos pesquisadores, permitindo que sejam alcançadas populações específicas, além de levar à redução dos custos (Wachelke et al., 2014). Apesar disso, tem-se observado que pesquisas de levantamento de dados através da internet tendem a apresentar taxas de participação menores do que as que utilizam métodos tradicionais (Bech \& Kristensen, 2009). 
Após o vencimento do prazo máximo estipulado para preenchimento do formulário online, iniciou-se o período de análises dos resultados obtidos. Inicialmente foi contabilizado o número total de respostas obtidas em cada categoria (discentes, docentes e técnicos), seguida da avaliação do número de representantes de cada área (ciências humanas, exatas, naturais e da saúde) em cada categoria. Foi obtido o escore total de cada participante e, a partir disso, foi feita a média e o desvio padrão dos escores da amostra de cada categoria e de cada área. O próximo passo foi a realização da análise estatística dos dados com auxílio do teste estatístico ANOVA para a comparação entre as médias dos escores entre os grupos. Para isso foi utilizado o programa estatístico GraphPrism 5.0, utilizando o teste de correlação de Pearson, considerando como nível de significância $\mathrm{p}<0,05)$.

Os participantes que obtiveram resultados significativos a partir da comparação estatística entre as categorias e áreas, foram encaminhados para que seja feito o agendamento do aconselhamento psicológico e psiquiátrico com os profissionais colaboradores da pesquisa.

\section{Resultados}

A média de idade total foi de $28,81 \pm 10,77$ anos, sendo que na área de biológicas foi de $26,07 \pm 8,25$ anos; 30,21 $\pm 11,95$

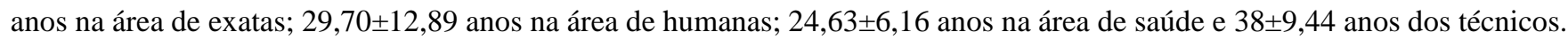

Ao analisar separadamente o total de indivíduos avaliados foi observado um valor de 27 mulheres na área de biológicas, 25 mulheres na área de exatas, 35 mulheres na área de humanas, 54 mulheres na área da saúde e 22 mulheres que são técnicas. Foram avaliados 16 homens na área de biológicas, 27 homens na área de exatas, 28 homens na área de humanas, 19 homens na área da saúde, e 10 técnicos.

Os dados do presente estudo demonstraram diferenças entre as médias da escala entre as classes estudadas: discentes, docentes e técnicos e entre as áreas dos discentes e docentes. Foram avaliados 178 discentes com média de idade de 22,9 \pm 4,46; 53 pontos dos docentes com média de idade de 43,09 $\pm 9,17$ pontos e 32 técnicos com média de idade de 38,00 $\pm 9,44$ pontos (Média \pm Desvio-padrão).

Os resultados da média do escore da escala entre os discentes demonstraram valores de 39,52 $\pm 30,17$ pontos na área de saúde; 49,48 $\pm 33,39$ pontos na área de exatas; $53,95 \pm 39,53$ pontos na área de humanas e 46,28 $\pm 30,61$ pontos na área de biológicas. A análise estatística demonstrou que ocorreu diferença significativa entre as médias dos escores nas áreas dos discentes com valor de $\mathrm{p}=0,05$ e F=3,056, como demonstrado na Figura 1.

Figura 1. Média dos escores da escala entre os discentes nas áreas de: biológicas, exatas, humanas e saúde.

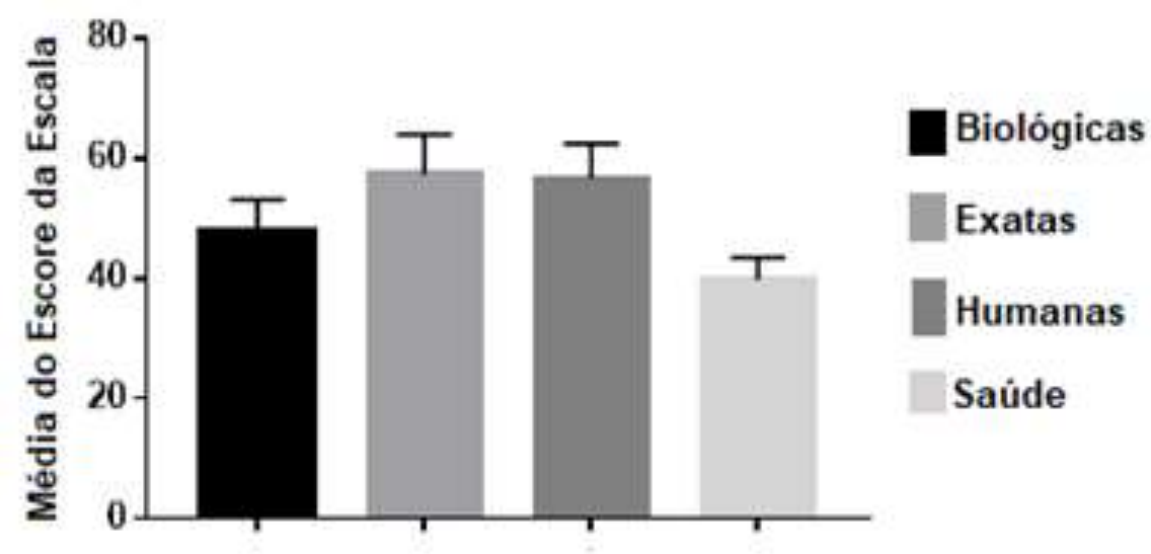

Fonte: Autores. 


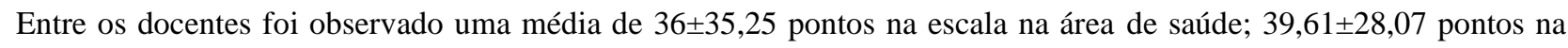

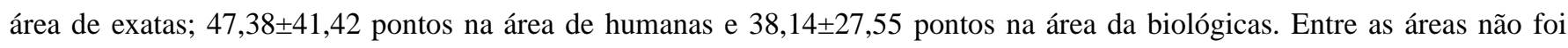
observada diferença significativa com valor de $\mathrm{p}=0,0905$ e $\mathrm{F}=2,186$.

Ao analisar a média total do escore da escala entre as três profissões foi observada uma média e desvio padrão entre os discentes de 48,52 $\pm 34,22$ pontos $(n=178)$; entre os docentes de 41,72 $\pm 33,1$ pontos $(n=53)$ e entre os técnicos de $42,38 \pm 34,96$ pontos $(n=32)$. Os dados demonstraram que não ocorreu diferença significativa entre as médias dos escores entre as três classes, com valor de $\mathrm{p}=0,3457$ e $\mathrm{F}=1,067$.

Ao analisar separadamente os escores em intervalos de 0-39, 40-79, 80-119, 120-159 e 160-199 pontos na escala foram evidenciados os seguintes dados da Tabela 4.

Tabela 4. Escores dos indivíduos em intervalos e área de atuação.

\begin{tabular}{lcrrrrr}
\hline Parâmetro & Biológicas & Exatas & Humanas & Saúde & Técnico & \multicolumn{1}{c}{ Total } \\
\hline Escores entre 0-39 & 24 & 26 & 30 & 45 & 19 & 144 \\
Escores entre 40-79 & 12 & 15 & 17 & 19 & 9 & 72 \\
Escores entre 80-119 & 6 & 10 & 11 & 7 & 3 & 37 \\
Escores entre 120-159 & 1 & 1 & 4 & 2 & 0 & 8 \\
Escores entre 160-204 & 0 & 0 & 1 & 0 & 1 & 2 \\
\hline
\end{tabular}

Fonte: Dados coletados na pesquisa.

\section{Discussão}

O presente estudo possibilitou a identificação de apenas alguns aspectos clínicos da SA, mas é preciso ressaltar que, para a obtenção do diagnóstico definitivo, é necessário que outros testes e avaliações psicológicas e psiquiátricas sejam utilizados. Em sua tese de mestrado, Caires (2012) aponta que, para o diagnóstico de SA é necessário mais de uma fase de avaliação, podendo envolver inicialmente a Escala Australiana que identifica comportamentos sugestivos de SA, mas que também é necessário o uso de outros meios para a obtenção do diagnóstico de forma fidedigna. Essas outras fases devem testar as capacidades sociais, emocionais, de concentração e cognitivas, além de avaliar aspectos específicos relacionados aos interesses do paciente, questionários, relatórios das instituições por ele frequentadas e dos profissionais com quem faz acompanhamento (Caires, 2012).

Os dados apontaram uma maior participação das mulheres na pesquisa. Isto pode ser um indicativo da maior adesão das mesmas ao questionário, mas também ajuda a corroborar os resultados de estudos que apontam a maior participação das mulheres no meio acadêmico (Bolzani, 2017). No Brasil, a representação desigual das mulheres é um fenômeno em movimento e vem se alterando rapidamente na base da pirâmide educacional. De acordo com o censo escolar do Instituto Nacional de Estudos e Pesquisas Educacionais Anísio Teixeira (Inep), o número de mulheres que concluiu o ensino médio é ligeiramente superior ao de homens no período de 2000 a 2012. Nos cursos de graduação, considerando-se todas as carreiras, aí incluídas áreas onde a predominância feminina é marcante, como pedagogia, letras, ciências humanas, em 2012, elas representavam 57,1\% dos concluintes (Bolzani, 2017).

A SA se distingue do autismo, principalmente, pela ausência de prejuízos nas áreas de linguagem e cognição (Brito et al., 2015). No presente estudo, quando realizada a comparação entre os três diferentes cargos institucionais, foi observado que os maiores escores foram encontrados entre os discentes, apontando uma maior prevalência de aspectos clínicos de SA nesse grupo. Os menores escores foram identificados entre os docentes. Tais profissionais possuem o papel de instruir e estimular o estudante na tomada de decisões, a fazer observações, perceber relações e trabalhar com indagações (Bettancourt et al., 2011). 
Assim, a docência exige níveis de habilidades sociais para interação e didática educacional que geralmente estão limitados em indivíduos com SA.

A habilidade para cálculos matemáticos complexos e a tendência à interpretação literal, que inclui incapacidade para interpretar metáforas e ironias (Brito et al., 2015), acabam aproximando as pessoas com SA da área de exatas. No presente estudo foi observado maiores médias dos escores nas áreas de humanas e exatas. Entre os representantes do curso de humanas que participaram da pesquisa, observou-se que um maior número de respostas foi obtido nos cursos de geografia e ciências econômicas. Sabe-se que tais cursos possuem uma carga relevante de disciplinas da área de exatas, podendo ser esse o motivo da área de humanas também apresentar escores elevados nos resultados.

O curso de música também é caracterizado como sendo de humanas e sabe-se que pessoas com SA possuem uma hipersensibilidade sensorial, podendo ser atraídos pela música. Outra opção seria o viés do tamanho da amostra, que, apesar de suficiente para a realização de uma avaliação da instituição, é pequeno quando extrapolado para projeções maiores, sendo necessário portanto, que outras pesquisas desse tipo sejam realizadas, com amostras maiores.

Uma das principais características da SA é a dificuldade de interação social, bem como formar e manter relacionamentos tanto pela dificuldade da comunicação não verbal, quanto pelo menor contato visual ou a fala pouco animada (Brito et al., 2015). Tal fato justifica a menor média dos escores na área de saúde entre os discentes e docentes, uma vez que esta área do conhecimento exige maior interação social e a criação de vínculo por parte do profissional em relação ao paciente.

A SA é um transtorno do neurodesenvolvimento caracterizada por deterioração significativa nas áreas sociais, ocupacionais e outras importantes atividades do indivíduo, apesar de não haver nenhum atraso geral na linguagem ou atrasos clinicamente significativos no desenvolvimento cognitivo, nas habilidades de autoajuda específicas para a idade, no comportamento adaptativo e na curiosidade sobre o ambiente na infância (Brito et al., 2015). Os indivíduos portadores da SA, geralmente apresentam grande habilidade em memorização, são bons em matemática ou ciência e, mesmo que não apresentem qualquer atraso cognitivo ou no desenvolvimento da fala, é importante que recebam uma educação especializada o mais cedo possível (Laia, 2012). No estudo de Costa et al., (2021) foi relatado que o tratamento utilizando a Interface entre Cérebro e Computador (ICC) vem auxiliando na melhora da atenção, comportamento e interação social de crianças com TDAH.

Como foi possível observar no estudo, a maioria dos indivíduos apresentaram escores entre 0-79 pontos. Isto pode ser devido a diversas causas. A primeira a ser levantada seria realmente uma baixa prevalência desta síndrome na população em geral e também no ambiente universitário. Como relata Klin (2006), os índices são de cerca de mais quatro indivíduos com transtorno do espectro do autismo (e.g., SA, TID-SOE) a cada 1.000 nascimentos. A própria amostra corroborou com tais resultados epidemiológicos, tendo em vista que dos 263 participantes, apenas 2 ficaram com escores entre 160-204.

Outra hipótese seria a própria dificuldade de tais indivíduos ingressarem no meio universitário, devido a uma menor inclusão e atenção de portadores da síndrome no meio escolar. No estudo de Dias et al. (2021) aponta-se para as dificuldades na inserção de estudantes com DTAH no ensino superior no Brasil. Novos estudos são necessários para avaliar a prevalência dos critérios analisados e dos resultados dos escores na população de ensino fundamental e médio, para verificar se há uma tendência de queda na prevalência à medida que se progride para esferas superiores do ensino.

Ainda existem muitas controvérsias sobre o diagnóstico e tratamento da SA, que tende a ser sub diagnosticada. Ainda assim, quando há a confirmação do diagnóstico, isto ocorre durante a infância, de forma que as escalas encontradas para a sua confirmação assim como os estudos sobre a síndrome, são voltadas para essa faixa etária, levando a um aumento da subnotificação pela dificuldade de se concluir o diagnóstico durante as outras fases da vida. No estudo de Damasceno et al., (2021) é mencionado que existem distúrbios de aprendizagem específicos aos estudantes como o Transtorno do Espectro Autista (TEA) e o Transtorno do Déficit de Atenção com Hiperatividade (TDAH). Com isso, torna-se extremamente importante o estudo sobre a presença dos aspectos clínicos entre a comunidade universitária, para melhor orientação sobre as 
estratégias de tratamento e intervenção, além de novas estratégias de ensino-aprendizagem. De acordo com Paula e Peixoto (2019), o processo de ensino-aprendizagem entre alunos com autismo e professores ainda apresenta muitos problemas que necessitam ser sanados através de estratégias de ensino-aprendizagem que auxiliem este processo.

\section{Conclusão}

No presente estudo foram evidenciados mais aspectos clínicos para SA entre os discentes, sendo mais prevalentes as áreas de humanas e exatas. No entanto, ao analisar os escores totais foram evidenciados que a maioria dos indivíduos avaliados se encontra entre os intervalos mais baixos da escala, demonstrando que essa população apresenta poucos aspectos clínicos para SA.

Sugere-se que novos estudos possam ser desenvolvidos com o intuito de investigar quais os fatores envolvidos com a maior média nos escores da escala dos aspectos clínicos da síndrome de Asperger entre os acadêmicos das áreas de humanas e exatas.

\section{Referências}

Bech, M. \& Kristensen, M. B. (2009). Differential response rates in postal and Web-based surveys in older respondents. Survey Research Methods, 3(1),1-6.

Bettancourt, L., Munoz, L. A., Merighi, M. A. B. \& Santos, M. F. (2011). O docente de enfermagem nos campos de prática clínica: um enfoque fenomenológico. Revista Latino-Americana Enfermagem. 19(5), 1-8.

Bolzani, V. S. (2017). Mulheres na ciência: por que ainda somos tão poucas? Ciência e Cultura, 69(4), 56-59.

Brito, A. P. L., Neto, A. R., Amaral, L. T., Balestra, R. L., Gonçalves, A. S. \& Castro, U. R. (2015). Síndrome de Asperger: Revisão de Literatura. Revista de Medicina e Saúde de Brasília. 169-76.

Brito, P. R., Rodriguez, A. R. P., Sosa, A. A. D., Gorrin, N. B., Martinez, A. V. C. M., Quintana, L. V., Díaz, B. C., Trujillo, C. A., González, P. M., Arévalo, C. S. L. de, López, K. M. F., Abellá, D. H., León, A. I. I. \& Rodríquez, M. I. R. (2011). Síndrome de Asperger en Atención Primaria: planificación de los cuidados. ENE, Revista Enfermería. 5(1), 90-9.

Caires, M. A. R. (2012). A importância da expressão dramática no desenvolvimento da comunicação e interação da criança com Síndrome de Asperger. Dissertação (Mestrado em Ciências da Educação na Especialidade em Domínio Cognitivo-Motor) - Escola Superior de Educação João de Deus. Lisboa, 180.

Costa, N. M. G. B., Marçal, E., Carvalho, M. M. \& Barbosa, T. C. S. (2021). Uso de interfaces cérebro-computador em crianças com TDAH: Uma revisão sistemática. Research, Society and Development. 10(7), e51110716929.

Damasceno, G. C., Cabral, D. A. C., Campos, F. M. S., Medeiros, I. P. V., Cabral, L. G. C. \& Cabral, K. M. C. (2021). As contribuições da neurociência à pedagogia: um diálogo necessário. Research, Society and Development. 10(1), e33710111846.

Diagn, M. (2013). Diagnostic and Statistical Manual of Mental Disorders - DSM V. 5th ed. Washington: American Psiquiatric Association.

Dias, S. (2015). Asperger e sua síndrome em 1944 e na atualidade. Rev. latinoam. psicopatol. fundam. 18(2), $307-313$.

Dias, M. A. M. B., Rosa, R. B., Pedroso, L. V., Pessano, E. F. C. \& Dinardi, A. J. (2021). Teaching methodologies and the inclusion promotion of students with Attention Deficit Hyperactivity Disorder (ADHD): An analysis in CAPES dissertations and theses. Research, Society and Development. 10(9), e18310917840.

Eckel, E., Schneider, G. N. \& Menezes, C. (2015). Síndrome de Asperger: visão geral e aspectos educacionais. Rev Maiêutica. 3(47), 69-75.

Estrela, C. (2018). Metodologia Científica: Ciência, Ensino, Pesquisa. Editora Artes Médicas.

Franz, L., \& Dawson, G. (2019). Implementing early intervention for autism spectrum disorder: a global perspective. Pediatric medicine. 2 , 44.

Klin, A. (2006). Autism and Asperger syndrome: an overview. Rev. Bras. Psiquiatr. 28(1), s3-s11.

Laia, J. D. L. (2012). Relatório de estágio profissional. 2012, 250f. Dissertação (Mestrado em Ensino dos $1 .^{\circ}$ e $2 .^{\circ}$ Ciclos do Ensino Básico Estágio Profissional I, II, III e IV) - Escola Superior de Educação João de Deus. Lisboa.

Paula, J. B., \& Peixoto, M. F. (2019). A inclusão do aluno com autismo na educação infantil: desafios e possibilidades. Cadernos da Pedagogia, 13(26), 3145.

Pereira A. S., Shitsuka, D. M., Parreira, F. J. \& Shitsuka, R. (2018). Metodologia da pesquisa científica. UFSM.

Teodoro, M. C., Casarini, K. A. \& Scorsolini-Comin, F. (2013). Intervenções terapêuticas em pessoas com Síndrome de Asperger: revisão da literatura. Barbaroi. 38, 6-25.

Wachelke, J., Natividade, J., Andrade, A., Wolter, R.\& Camargo, B. (2014). Caracterização e Avaliação de um Procedimento de Coleta de Dados Online (CORP). Aval. psicol. 13(1), 143-146. 